\title{
Degraded Image Enhancement Using Dual-Domain-Adaptive Wavelet and Improved Fuzzy Transform
}

\author{
Weiqiang Fan $\left(D,{ }^{1}\right.$ Yuehua Huo ${ }^{D}{ }^{2}$ and Xiaoyu $\mathrm{Li}^{1}$ \\ ${ }^{1}$ School of Mechanical Electronic and Information Engineering, China University of Mining and Technology, \\ Beijing 100083, China \\ ${ }^{2}$ Network and Information Center, China University of Mining and Technology, Beijing 100083, China \\ Correspondence should be addressed to Yuehua Huo; huoyh@cumtb.edu.cn
}

Received 7 January 2021; Revised 21 February 2021; Accepted 8 March 2021; Published 25 March 2021

Academic Editor: Xin Ma

Copyright (c) 2021 Weiqiang Fan et al. This is an open access article distributed under the Creative Commons Attribution License, which permits unrestricted use, distribution, and reproduction in any medium, provided the original work is properly cited.

\begin{abstract}
A novel enhancement algorithm for degraded image using dual-domain-adaptive wavelet and improved fuzzy transform is proposed, aiming at the problem of surveillance videos degradation caused by the complex lighting conditions underground coal mine. Firstly, the dual-domain filtering (DDF) is used to decompose the image into base image and detail image, and the contrast limited adaptive histogram enhancement (CLAHE) is adopted to adjust the overall brightness and contrast of the base image. Then, the discrete wavelet transform (DWT) is utilized to obtain the low frequency sub-band (LFS) and high frequency sub-band (HFS). Next, the wavelet shrinkage threshold is applied to calculate the wavelet threshold corresponding to the HFS at different scales. Meanwhile, a new Garrate threshold function that introduces adjustment factor and enhancement coefficient is designed to adaptively de-noise and enhance the HFS coefficients, and the Gamma function is employed to correct the LFS coefficients. Finally, the PAL fuzzy enhancement operator is improved and used to perform contrast enhancement and highlight area suppression on the reconstructed image to obtain an enhanced image. Experimental results show that the proposed algorithm can not only significantly improve the overall brightness and contrast of the degraded image but also suppresses the noise of dust and spray and enhances the image details. Compared with the similar algorithms of STFE, GTFE, CLAHE, SSR, MSR, DGR, and MSWT algorithms, the indicator values of comprehensive performance of the proposed algorithm are increased by $205 \%, 195 \%$, $200 \%, 185 \%, 185 \%, 85 \%, 140 \%$, and $215 \%$, respectively. Moreover, compared with the other seven algorithms, the proposed algorithm has strong robustness and is more suitable for image enhancement in different mine environments.
\end{abstract}

\section{Introduction}

With the increasing contradiction between mine safety production and economic benefits, the related safety issues have aroused wide concern of the country and society $[1,2]$. The processing and analysis of the intelligent monitoring video in mine is a necessary prerequisite for studying the location and status of moving objects such as underground personnel, vehicles, and equipment [3-5]. However, the images of surveillance videos are susceptible to dust and spray, low illumination or uneven lighting of artificial light source, and other environmental factors, resulting in poor image quality captured by the camera $[6,7]$. This directly affects the mine dispatch center's accurate grasp of the actual underground situation and subsequent data analysis. Therefore, in order to better present the scene information of coal mine, improve the visual effect of the image, and promote the application of the underground video monitoring system in the coal mine safety production and intelligent analysis, it is of great significance to study the enhancement of the degraded image in coal mine.

At present, some scholars at home and abroad have studied the problem of image enhancement under uneven lighting or low illumination conditions, but few of them have studied the problem of degraded image enhancement under complex lighting conditions in coal mine. As described in [7-9], the uneven lighting image enhancement based on retinex theory is realized, but these methods have problems 
such as local blocking effects, feature loss, and nonlinear distortion, which will result in the blurred edge features. Meanwhile, these kinds of methods has the following shortcomings: (1) The traditional filter estimates irradiation component (IC), and it can easily cause the edge feature of the enhanced image to be blurred; (2) the edge preserving filter estimates IC, but the enhancement process takes a long time; (3) the algorithm needs to assume that the ambient light is uniform, which is not suitable for the artificial light scene under the mine. DWT is an important image enhancement method, which has better time-frequency local characteristics and multiresolution analysis characteristics $[10]$. In $[11,12]$, a enhancement method using DWT and SVD is proposed, SVD is applied to DWT's LFS coefficients, and inverse DWT is used to reconstruct the processed HFS and LFS coefficients. Although the improvement effect is good, the brightness and SNR of the enhanced image are small. The DWT transform domain-based gamma correction proposed in $[13,14]$ have obvious enhancement effect on the degraded images under complex lighting conditions. However, the wavelet threshold function can easily lead to the loss of image details and the improvement of contrast is not significant; in addition, the process of manually adjusting gamma value is tedious. Singh et al. [15] proposed a piecewise gamma-corrected histogram equalization framework using particle swarm optimization (PSO) by analyzing the advantages and disadvantages of histogram and gamma transformation. This algorithm has a better enhancement effect on degraded images, but the process of parametric iterative optimization takes a long time and easy to fall into the local optimal solution.

For the drawbacks of the above existing algorithms, this paper proposes a method for degraded image enhancement that combines dual-domain-adaptive wavelet and improved fuzzy transform, in which a Garrate threshold function that introduces adaptive adjustment factor and enhancement coefficient is designed, and an improved PAL fuzzy transform function is proposed. First, the degraded image is decomposed into base image and detail image by DDF, base image represents the overall information of the original image, and detail image represents the detailed information of the original image. At the same time, the CLAHE is adopted to adjust the brightness and contrast of the base image. Then, the Garrate threshold function is designed to adaptively de-noise and enhance the HFS coefficients of the detail image. Meanwhile, the LFS coefficients are adjusted by the gamma transformation. Finally, the improved PAL fuzzy transformation method is used to suppress the halo and adjust the highlight area of the reconstructed image, and then the enhanced image is obtained. The rest of the paper is organized as follows. The basic principle of the proposed algorithm is described in Section 2. The implementation process of the algorithm is described in Section 3. The experimental results and analysis are discussed in Section 4. Some conclusions are summarized in Section 5.

\section{Image Enhancement Algorithm Modeling}

The DWT can transform the image from spatial domain to frequency domain, without losing the original information of the image and increasing redundant information [16]; moreover, it has perfect reconstruction ability $[17,18]$. However, the existing two-dimensional wavelet threshold function and threshold selection model have limited application scope. For complex lighting scenes in mine, traditional wavelet enhancement algorithms are easy to lose image detail features in HFS or amplify image noise, and unprocessed or poorly processed LFS coefficients contain image outline features. As a result, images after wavelet reconstruction (WR) appear different degrees of blur. Therefore, it is necessary to design an image enhancement model that meets the complex lighting conditions for the mine.

2.1. Wavelet Enhancement Model Design. The construction of wavelet threshold function is the key to the design of enhancement model. At present, the image enhancement algorithm using wavelet transform mainly uses traditional wavelet hard threshold function, soft threshold function $[19,20]$, and improved wavelet threshold function models: semisoft threshold function [21] and Garrote threshold function [22]. In view of the defects of the four typical wavelet threshold functions in different degrees [23] and the characteristics that the noise signal in HFS decreases gradually with the increase of Wavelet decomposition (WD) scale [24], a Garrote threshold function including adaptive factor is designed (as shown in Figure 1). This function is defined as follows:

$$
H\left(\omega_{i, j}\right)= \begin{cases}\operatorname{sgn}\left(\omega_{i, j}\right) \frac{\left(1-s_{i}\right)}{T_{i}} \omega_{i, j}^{2}, & \left|\omega_{i, j}\right|<T_{i}, \\ \omega_{i, j}-s_{i} \frac{T_{i}^{2}}{\omega_{i, j}} e^{T_{i}-\left|\omega_{i, j}\right|}, & \left|\omega_{i, j}\right| \geq T_{i},\end{cases}
$$

where $H$ is the HFS coefficient after wavelet de-noising; $s_{i}$ is an adaptive factor at the $i$ th scale, $s_{i} \in(0,1) ; \omega_{i, j}$ is the $j$ th HFS of the $i$ th layer $\mathrm{WD}, j=1,2$, and 3 , respectively, corresponding to $\mathrm{HL}, \mathrm{LH}$, and $\mathrm{HH}$ sub-bands; $\operatorname{sgn}(\cdot)$ is a symbolic function; and $T_{i}$ is the wavelet threshold at the $i$ th scale.

In the model, $s_{i}$ can be dynamically adjusted according to the distribution of noise coefficients after $\mathrm{WD}$, which greatly improves the flexibility and practicability of the model. $s_{i}$ is obtained as follows [24]:

$$
s_{i}=\frac{m}{M},
$$

where $M$ is the length of the HFS at the $i$ th scale and $m$ is the number of frequencies that the HFS at the $i$ th scale is less than the threshold. 
Generally, the HFS after WD contains not only a large amount of detail information but also some noise information [15]. If the wavelet threshold function of Equation (1) is directly used for the enhancement of image detail information, the noise signal will also be amplified to the same extent, which will affect the visual effect of the image. Therefore, this paper introduces the adaptive adjustment factor and the adaptive enhancement coefficient that achieve adaptive noise reduction and enhancement of HFS coefficients at different scales. The adaptive wavelet threshold function is

$$
H^{\prime}\left(\omega_{i, j}\right)= \begin{cases}\operatorname{sgn}\left(\omega_{i, j}\right) \frac{(1-s)}{T} W_{i, j}\left(\omega_{i, j}\right)^{2}, & \left|\omega_{i, j}\right|<T, \\ W_{i, j} \omega_{i, j}-s \frac{W_{i, j} T^{2}}{\omega_{i, j}} e^{W_{i, j}}\left(T-\left|\omega_{i, j}\right|\right), & \left|\omega_{i, j}\right| \geq T,\end{cases}
$$

where $H^{\prime}$ is the HFS coefficient after wavelet de-noising and enhancement and $W_{i, j}$ is the adaptive enhancement coefficient of the $j$ th HFS under the $i$ th scale.

The scale of WD is negatively correlated with the detailed information in the corresponding HFS [15]. Therefore, $W_{i, j}$ can be obtained by the following equation:

$$
W_{i, j}=k e^{\left(\omega_{i, j}-\mu\right)^{2} / 2 \sigma^{2}},
$$

where $\mu$ and $\sigma$ are the mean and standard deviation of the $j$ th HFS under the $i$ th scale, respectively, and $K$ is an adaptive adjustment parameter.

After WD, the HH subimage at the same scale contains more details of the original image, while the HL and LH subimages have less detailed features and larger approximate features [25]. Therefore, when $j=1,2, k=2 i-1$; when $j=3$, $k=2 i$.

According to Equations (3)-(4), the adaptive wavelet threshold function designed is continuous and smooth in the whole definition domain; there is no constant deviation. On the basis of the change of WD scale, it can perform adaptive threshold transformation for HFS at each scale, highlight the detailed features of different scales, suppress the noise level of the image, enhance the layering of the image, and greatly improve the flexibility and practicality of the wavelet threshold function.

2.2. Threshold Selection. In image enhancement processing based on DWT, the selection of wavelet threshold is a decisive factor in determining the image enhancement effect. As the scale of WD increases, the noise coefficient in the corresponding HFS will become smaller and smaller [26]. The same threshold is used at different scales, if the threshold is selected too large, the effective wavelet coefficient below the threshold is set to zero, resulting in the blurring of image details and edge features; if the threshold is selected too small, the wavelet coefficients after de-noising still contains a lot of noise, which reduces the de-noising effect of the image enhancement algorithm. Therefore, this paper uses the wavelet shrinkage threshold method based on the Bayesian estimation to achieve adaptive adjustment of the wavelet threshold. The specific calculation process is as follows:

(1) According to the theory of Bayesian estimation, each HFS coefficient after wavelet de-noising obeys a generalized Gaussian distribution with a mean of 0 and a variance of $\sigma_{x}^{2}$ [27]:

$$
\Phi(x)=\frac{1}{\sqrt{2 \pi \sigma_{x}^{2}}} \exp \left(-\frac{x^{2}}{2 \sigma_{x}^{2}}\right),
$$

where $x$ and $\sigma_{x}$ are the jth HFS coefficient and standard deviation at the $i$ th scale.

(2) When the parameter $\sigma_{x}$ is given, the optimal threshold $T$ can be found according to the Bayesian risk estimation function $r(T)$ [28]. The threshold is obtained as follows [29]:

$$
T_{i}=\frac{\sigma^{2}}{\sigma_{x}},
$$

where $T_{i}$ is the threshold of the HFS coefficient of the $i$ th scale and $\sigma^{2}$ is the noise variance of the $j$ th HFS coefficient of the $i$ th layer. In noise estimation, $j$ is usually set to 3 .

(3) The noise variance is calculated using the robust median estimation [30]:

$$
\sigma=\frac{\operatorname{median}\left(\left|\omega_{i, j}\right|\right)}{0.6745} .
$$

(4) The variance estimate of each noisy observation subband is obtained by the maximum likelihood estimation method [24]:

$$
\sigma_{y}^{2}=\frac{1}{n} \sum_{x=1}^{n} \omega_{i, 3}^{2}(x)
$$

where $n$ is the length of the HH sub-band under the $i$ th layer.

(5) Obtained by $\sigma_{y}^{2}=\sigma_{x}^{2}+\sigma^{2}$ :

$$
\sigma_{x}=\sqrt{\max \left(\sigma_{y}^{2}-\sigma^{2}, 0\right)} .
$$

The adaptive wavelet threshold $T$ at different wavelet scales is calculated by Equations (6)-(9).

2.3. Gamma Transform. The low brightness of the mine degraded image is easy to cause the LFS coefficients after WD to be small as a whole, which makes the image contour characteristics after WR not obvious. So, the gamma transform in this paper is used to correct LFS coefficients, highlight the contour characteristics of the reconstructed image, and improve the overall brightness of the degraded image. Gamma transform is completed by the following equation [31]: 


$$
\widehat{f}_{\mathrm{LFS}}(x, y)=\left(\frac{f_{\mathrm{LFS}}(x, y)-f_{\mathrm{LFS}, \min }(x, y)}{f_{\mathrm{LFS}, \max }(x, y)-f_{\mathrm{LFS}, \text { min }}(x, y)}\right)^{(1 / \varepsilon)} *\left(f_{\mathrm{LFS}, \max }(x, y)-f_{\mathrm{LFS}, \text { min }}(x, y)\right)+f_{\mathrm{LFS}, \text { min }}(x, y),
$$

where $\hat{f}_{\text {LFS }}(x, y)$ is the LFS coefficients after gamma transformation; $f_{\mathrm{LFS}}(x, y)$ is the LFS coefficients; $f_{\mathrm{LFS}, \text { max }}$ $(x, y)$ is the maximum value of the LFS coefficients; $f_{\mathrm{LFS} \text {,min }}$ $(x, y)$ is the minimum value of the LFS coefficients; and $\varepsilon$ is an adjustable parameter, which is used to control the degree of enhancement or suppression of image brightness.

In general, the larger the $\varepsilon$, the greater the degree of enhancement, the more obvious the brightness increase. The smaller the $\varepsilon$, the greater the degree of suppression, the more obvious the brightness suppression. As the brightness of the mine degraded image is overall dark and only part of the area has higher brightness, the $\varepsilon$ for mine image enhancement is generally set to 2.5 .

2.4. Improved PAL Fuzzy Enhancement Algorithm. After image reconstruction, the brightness and contrast of mine degraded images have been improved to a certain extent, but the effect of suppressing the image halo caused by artificial light sources is not obvious. In order to further enhance the detailed features of the degraded image and suppress its image halo, an improved PAL fuzzy enhancement algorithm is designed to achieve blur enhancement of the reconstructed image. The specific steps are as follows:

(1)Design a membership function:

$$
Y_{m, n}=\frac{X_{m, n}-X_{\min }}{D\left(X_{\max }-X_{\min }\right)}, \quad 1 \leq D \leq 2,
$$

where $X_{\max }=\max \left\{X_{m, n}\right\}$ and $X_{\min }=\min \left\{X_{m, n}\right\}$ are the maximum and minimum values of all pixels in the image after WR and $D$ is the control parameter, $D \in[1,2]$.

(2) Calculate the membership of the elements in the reconstructed image by Equation (11), and perform the fuzzy operation through the constructed fuzzy enhancement operator. Fuzzy enhancement operator:

$$
Y_{m, n}^{\prime}=\left\{\begin{array}{l}
\left(2 Y_{m, n}\right)^{(1 / 2)}, \quad 0 \leq Y_{m, n}<0.5 \\
2 Y_{m, n}^{2}-Y_{m, n}+1, \quad Y_{m, n} \geq 0.5
\end{array}\right.
$$

(3) Inverse fuzzy transformation is performed on the fuzzy enhancement coefficient obtained by Equation (12). Antifuzzy transform function:

$$
f_{E}=D\left(X_{\max }-X_{\min }\right) \frac{Y_{m, n}^{\prime}}{2}+X_{\min }
$$

The improved PAL fuzzy enhancement algorithm uses a membership function containing control parameters $D$ to transform the image from the spatial domain to the fuzzy set domain. According to the designed fuzzy enhancement operator, the image halo suppression and the low illumination area enhancement are performed, and the final enhanced image is obtained by the antifuzzy transformation function. By adjusting the value of $D$, the membership of the gray values of different degraded images is controlled, and then the video surveillance images in different lighting areas of the mine are enhanced, which greatly improves the robustness of the proposed algorithm.

\section{Implementation Process of the Proposed Algorithm}

This paper proposes the enhancement algorithm for degraded image using dual-domain-adaptive wavelet and improved fuzzy transform. The specific implementation steps can be concluded as follows:

Step 1. Perform DDF [32] on the 3-channel of the degraded image $f$ (i.e., $R, \mathrm{G}$, and $\mathrm{B}$ ) to obtain the base image and detail image of the three channels, respectively.

Step 2. CLAHE algorithm is used to adjust the illumination and contrast of the acquired 3-channel base image.

Step 3. Use the "db5" wavelet base [33] to perform WD of 3layer on the detail image to obtain LFS and HFS at each scale.

Step 4. Calculate the wavelet threshold $T$ and adaptive weighting factor $s$ corresponding to each scale HFS.

Step 5. Calculate the adaptive enhancement coefficient Wi,j for each scale HFS according to Equation (4).

Step 6. The adaptive weight factor $s$, adaptive enhancement coefficient $\mathrm{Wi}, \mathrm{j}$, and wavelet threshold $T$ are introduced into the adaptive wavelet threshold function of Equation (3) to achieve de-noising and enhancement of HFS at various scales.

Step 7. Gamma transformation is performed on the LFS coefficients, and WR is performed on each scale HFS after de-noising and enhancement and the LFS after gamma transformation.

Step 8. Repeat the Step $3 \sim$ Step 7 to obtain the 3-channel detail image after WR.

Step 9. Reconstruct the processed 3-channel base image and the 3-channel detail image after WR to obtain a reconstructed image.

Step 10. Convert the reconstructed image from the RGB color model to the HSV color model, and use the improved 

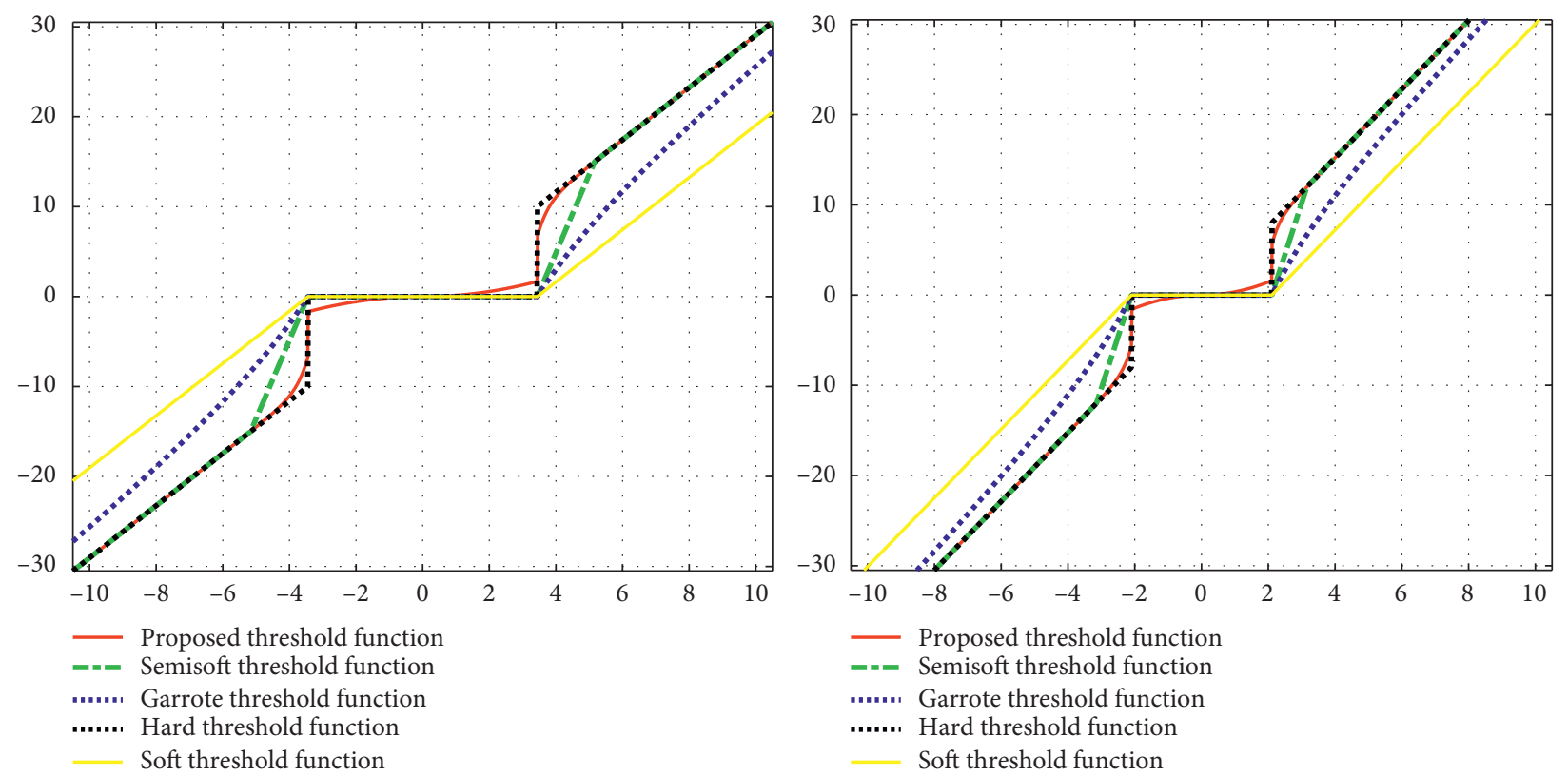

Figure 1: The proposed threshold function.

PAL fuzzy enhancement algorithm to blur the acquired V channel image, and obtain the final enhanced image.

The block diagram of the proposed algorithm implementation principle is shown in Figure 2.

\section{Experimental and Result Analysis}

In order to verify the practical application effect of the proposed algorithm, some degraded images in different mine monitoring videos were selected. Experimental computer configuration: Inter Core i7-10750H CPU, $2.60 \mathrm{GHz}$, RAM $16 \mathrm{~GB}$, programming tool: Matlab R2020a. The enhanced performance of the proposed algorithm and other seven comparison algorithms is analyzed from two aspects: subjective vision and objective indicators. The seven comparison algorithms are the Semisoft threshold function enhancement (proposed STFE), the Garrate threshold function enhancement (proposed GTFE), CLAHE [9], single scale retinex (SSR) [8], multiscale retinex (MSR) [34], double gamma retinex (DGR) [35], and contrast limited adaptive histogram equalization discrete wavelet transform (CLAHEDWT) [36]. The parameter settings in the above comparison algorithms are as follows: the proposed STFE and GTFE use DWT to de-noise and adjust the contrast of the image, and wavelet threshold uses a fixed threshold (sqtwolog), enhancement coefficient $W_{i, j}=2$, and other parameters are set as the same as the proposed algorithm. CLAHE uses the default parameters, and SSR uses Gaussian filter. MSR uses the default parameters of multiresolution McCann. DGR and CLAHE-DWT use the parameters recommended in the original reference.
4.1. Subjective Analysis. The experiment selected 17 images in 3 typical scenarios to test the proposed algorithm and seven comparison algorithms, including low illumination images, dust and spray images, and uneven lighting images. In view of the limited length of the article, this subsection only shows part of the experimental results after image enhancement:

(1) Experiment 1: the low illumination image enhancement experiment: The image resolution of the tunneling face is $355 \times 568$, and the enhanced results are shown in Figure 3.

(2) Experiment 2: the dust and spray image enhancement experiment: the image resolution of the fully mechanized mining face is $520 \times 920$, and the enhanced results are shown in Figure 4.

(3) Experiment 3: the uneven lighting image enhancement experiment: the image resolution of the auxiliary transportation tunnel is $520 \times 920$, and the enhanced results are shown in Figure 5.

Comparing the original images and the enhanced visual effect images (Figure 3-5), it can be seen that the degraded images have low brightness and contrast, severe dust and spray interference, and uneven brightness of artificial light sources. The enhancement effects of different algorithms are significantly different. The contrast of Figures $3(b)-5(b)$ and $3(c)-5(c)$ enhanced by the proposed STFE and GTFE is significantly improved, but the highlight area and halo phenomenon of the images have not been improved, and different degrees of blur appear. The brightness and contrast of the Figure 3(d)-5(d) enhanced by the CLAHE are 


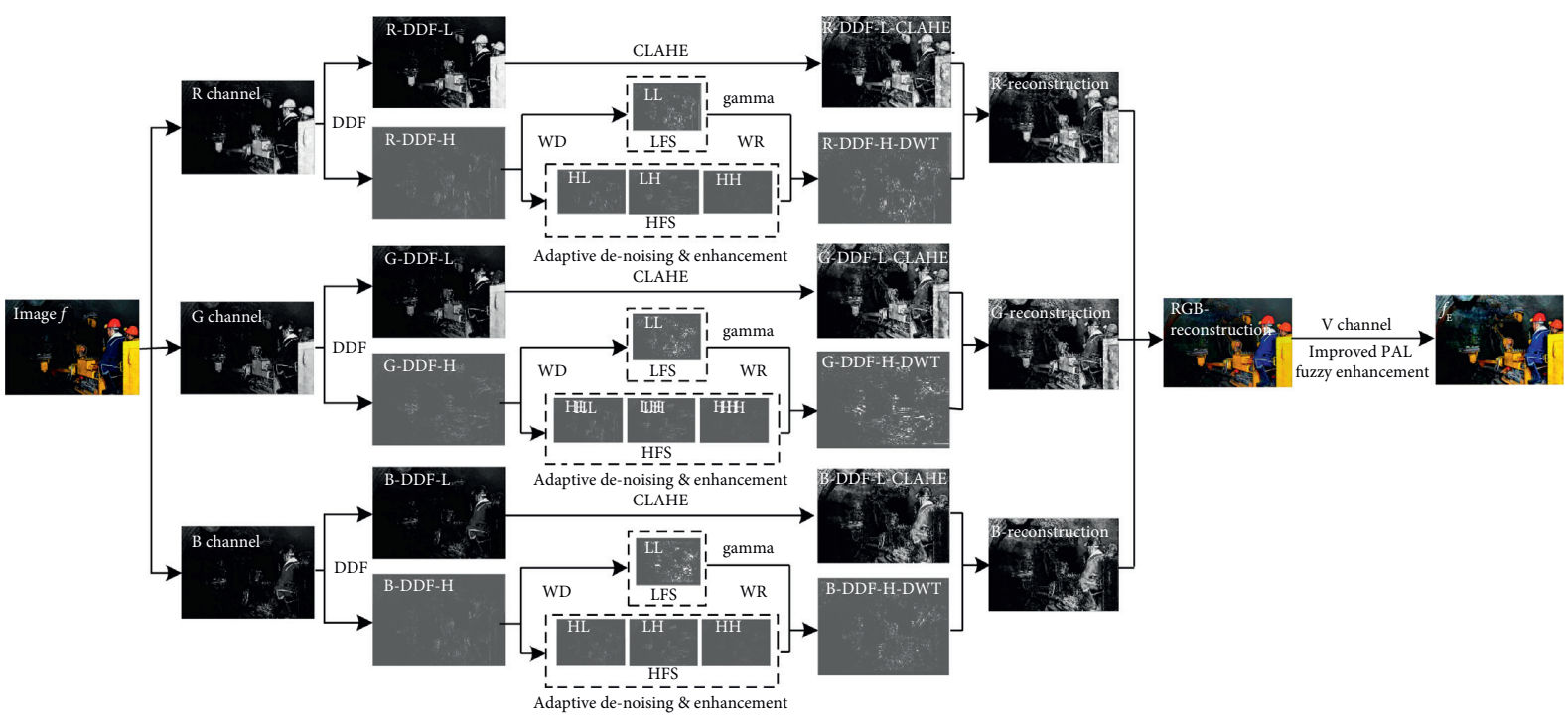

Figure 2: Block diagram of the proposed algorithm implementation principle.

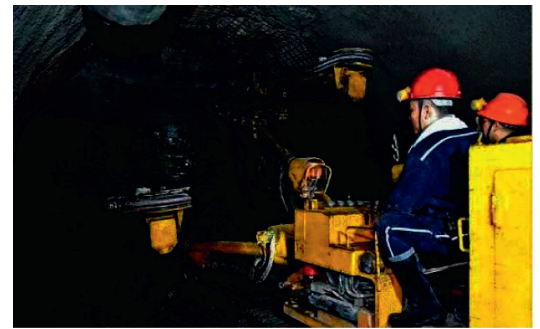

(a)

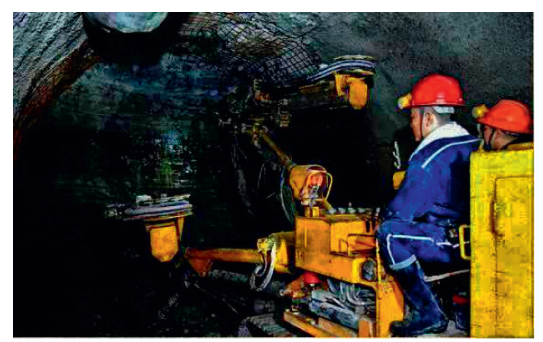

(d)

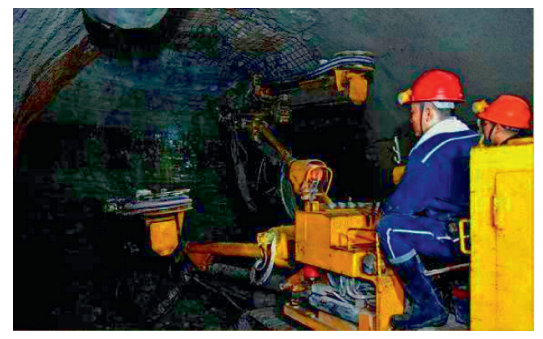

(g)

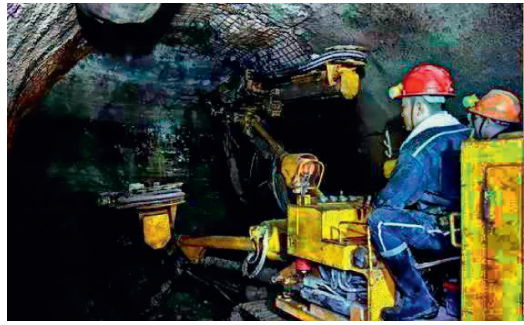

(b)

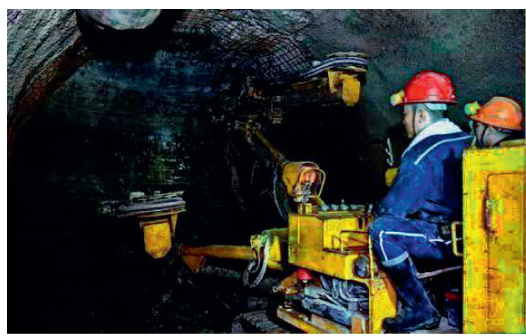

(e)

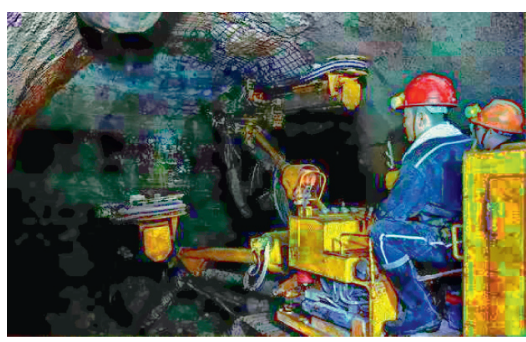

(h)

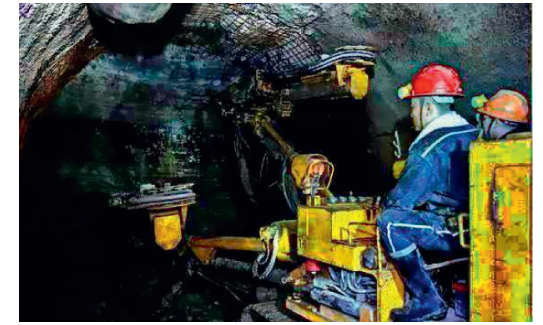

(c)

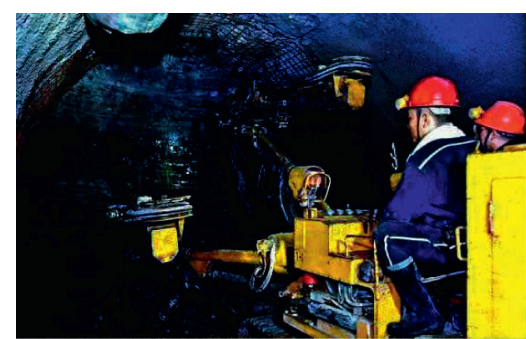

(f)

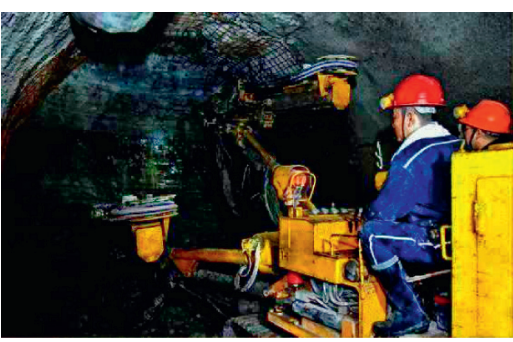

(i)

FIgURE 3: Comparison of the low illumination image enhancement effect. (a) Original image $f$, (b) proposed STFE, (c) proposed GTFE, (d) CLAHE, (e) SSR, (f) MSR, (g) DGR, (h) CLAHE-DWT, and (i) target algorithm.

improved to a certain extent, but the halo and highlight areas of the image are enhanced. Figures $3(\mathrm{e})-5(\mathrm{e})$ enhanced by SSR show over-enhancement phenomenon. Figures 3(f)5(f) enhanced by the MSR are better than STFE, GTFE, CLAHE, and SSR, but it also has color distortion. The brightness of the Figures $3(\mathrm{~g})-5(\mathrm{~g})$ enhanced by the DGR is significantly improved, but there is distortion in the dark areas of the images and seriously distorted in the uneven lighting environment in the mine. The brightness of the Figures 3(h)-5(h) enhanced by CLAHE-DWT is obviously 


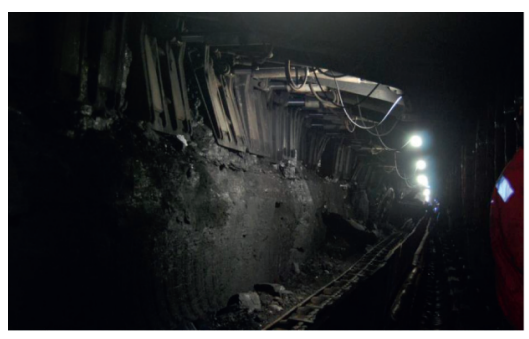

(a)

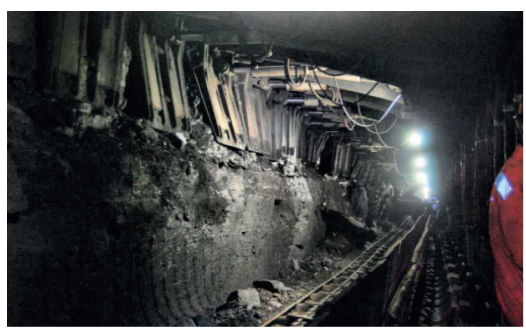

(d)

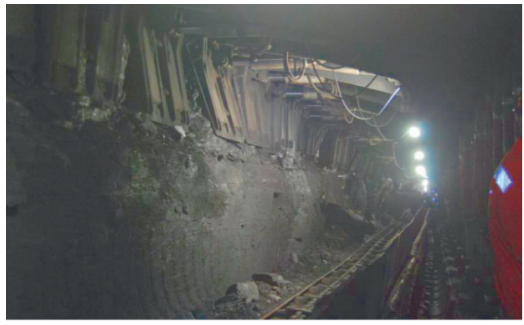

(g)

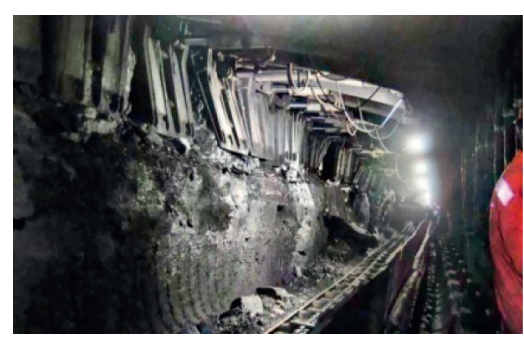

(b)

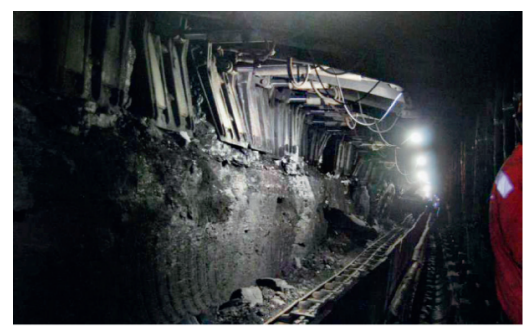

(e)

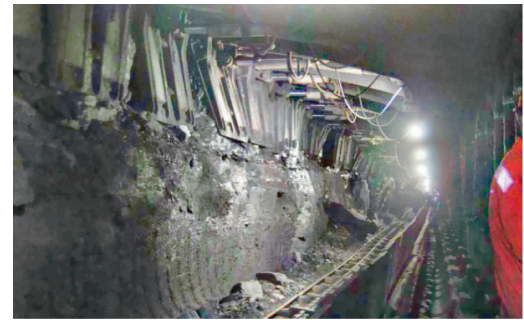

(h)

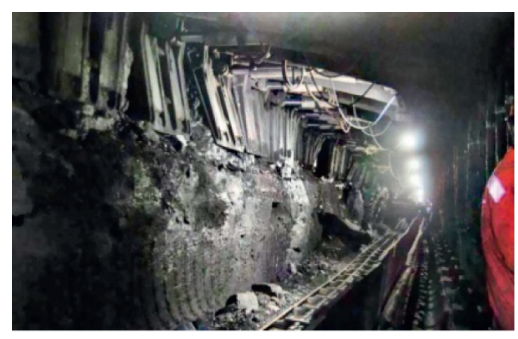

(c)

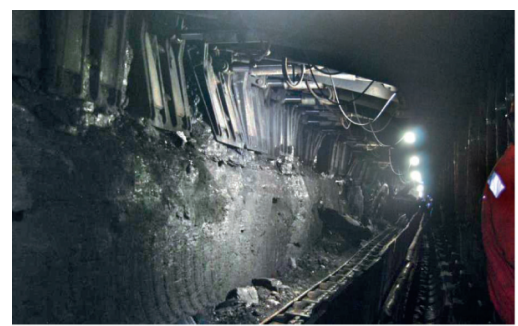

(f)

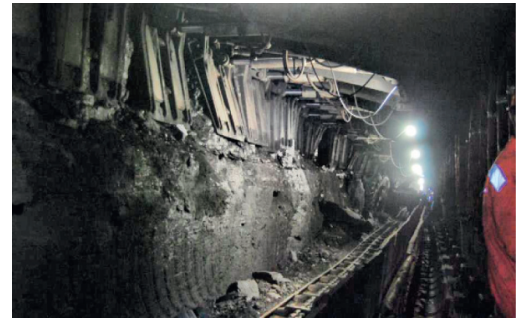

(i)

FIGURE 4: Comparison of the dust and spray image enhancement effect. (a) Original image $f$, (b) proposed STFE, (c) proposed GTFE, (d) CLAHE, (e) SSR, (f) MSR, (g) DGR, (h) CLAHE-DWT, (i) target algorithm.

improved, but the contrast of the images is reduced and the edges are blurred. For the above three scenarios, the images enhanced by the proposed algorithm have significant improvements in overall brightness, contrast, color, and edges, and the visual effect is better than the other seven contrast algorithms.

4.2. Objective Analysis. In order to objectively evaluate the effect of image enhancement, this paper adopts five indicators of Mean (image brightness) [15], mean local mean square error (MLMSE) (image contrast), peak signal to noise ratio (PSNR) (noise suppression level), mean local information entropy (MLIE) (information richness) [31], and structural similarity index measure (SSIM) (image distortion degree) [37] for performance evaluation and comparison. The objective metrics data of low illumination (Experiment 1), dust and spray (Experiment 2), and uneven lighting (Experiment 3) underground coal mine are shown in Tables 1-3.

The proposed algorithm and seven comparison algorithms were tested on the 17 images in three typical scenarios, and the average values of the objective metrics data are shown in Table 4.

According to the data in Tables 1-4, we know that the five indicator values of different algorithms have their own high and low values. In order to analyze the comprehensive enhanced performance of different algorithms more intuitively, the comprehensive indicator $F$ is used to calculate the cumulative value of normalization of the five indicators. Assuming that the PSNR and SSIM of the original image $f$ are 10 and 1 , respectively, then $F$ is shown in Table 5:

$$
F=\sum_{i=1}^{p} w_{i} \frac{x_{i}-x_{\min }}{x_{\max }-x_{\min }},
$$

where $x_{\max }$ is the maximum value of the five indicators; $x_{\min }$ is the minimum value of the five indicators; $x_{i}$ is the $i$ th indicator value of a certain algorithm; $p$ is the number of evaluation indicators, $p=5$; and $W$ is the weight. The indicators of mean, MLMSE, PSNR, MLIE, and SSIM in the paper are independent of each other, and $w=0.25$.

According to the comprehensive performance evaluation data under the three experimental conditions in Table 5, the line chart of the comprehensive indicator $F$ for all the algorithms is drawn as Figure 6.

According to the comprehensive evaluation data, graphs, and tables of Experiment 1, Experiment 2, Experiment 3, and 17 images in Table 4 and Figure 6, it can be seen that under three different experimental conditions, compared with other seven algorithms, the $F$ value of the proposed algorithm tends to be more stable, and the robustness is also 


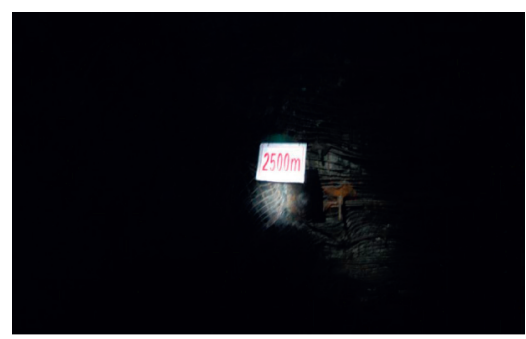

(a)

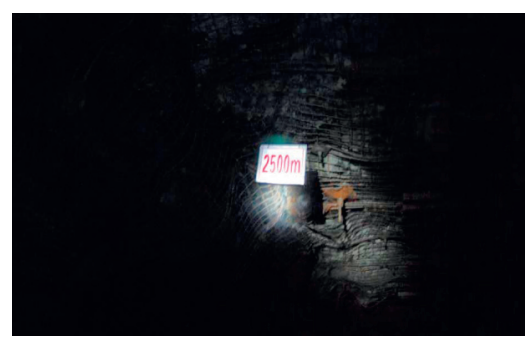

(d)

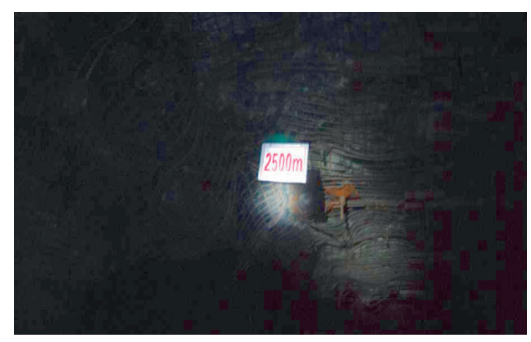

$(\mathrm{g})$

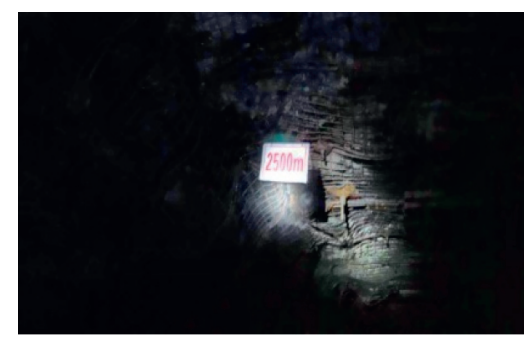

(b)

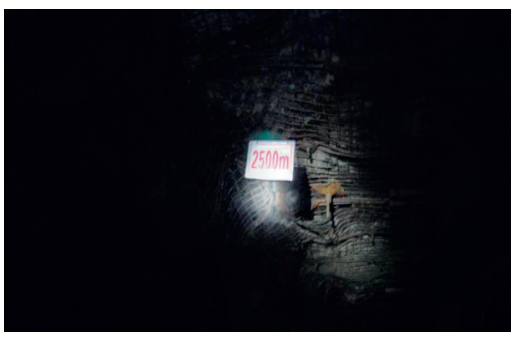

(e)

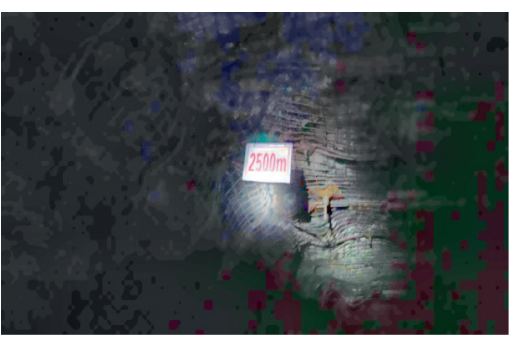

(h)

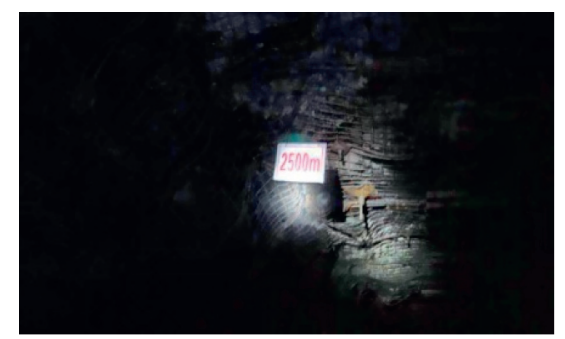

(c)

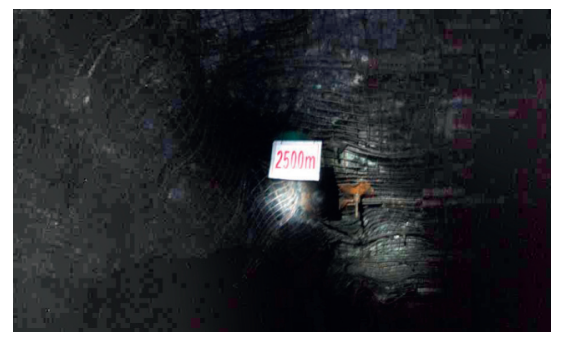

(f)

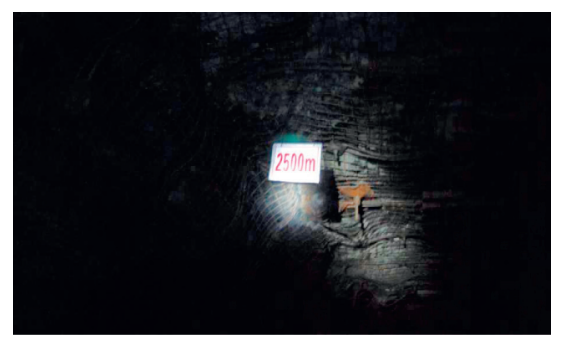

(i)

FIgURE 5: Comparison of the uneven lighting image enhancement effect. (a) Original image $f$, (b) proposed STFE, (c) Proposed GTFE, (d) CLAHE, (e) SSR, (f) MSR, (g) DGR, (h) CLAHE-DWT, (i) target algorithm.

TABLE 1: Evaluation indicator values of enhanced images under low illumination.

\begin{tabular}{|c|c|c|c|c|c|}
\hline Evaluation index & Mean & MLMSE & PSNR & MLIE & SSIM \\
\hline Original image $f$ & 34.91 & 226.52 & -- & 2.01 & - - \\
\hline Proposed STFE & 36.67 & 488.95 & 26.12 & 2.02 & 0.98 \\
\hline Proposed GTFE & 36.56 & 476.08 & 26.29 & 1.99 & 0.98 \\
\hline CLAHE & 52.26 & 402.29 & 19.12 & 2.15 & 0.85 \\
\hline SSR & 53.88 & 375.12 & 18.62 & 2.16 & 0.84 \\
\hline MSR & 47.23 & 423.26 & 21.68 & 2.09 & 0.94 \\
\hline DGR & 60.89 & 293.63 & 17.87 & 2.10 & 0.81 \\
\hline CLAHE-DWT & 88.52 & 401.94 & 11.86 & 2.18 & 0.53 \\
\hline Target algorithm & 59.15 & 405.68 & 17.35 & 2.25 & 0.80 \\
\hline
\end{tabular}

stronger. In the dust and spray environment, the proposed STFE has a higher $F$ value than the proposed algorithm, but in the low illumination and uneven lighting environment, the proposed algorithm has a better $F$ value than the proposed STFE and GTFE, which verifies that the proposed STFE and GTFE using DWT have excellent de-noising ability. In the dust and spray environment, the proposed algorithm can not only de-noise but also improve image contrast and increase detail information. According to the $F$ value of 17 images in three typical scenarios, it can be seen that the comprehensive performance of the proposed algorithm is better than the other seven comparison algorithms.

According to the $F$ value of the 17 images in Table 4, we plot the line chart, calculate the relative percentage of the proposed algorithm and seven comparison algorithms compared with the original image, and draw the histogram of the relative percentage, as in Figure 7.

According to the data in Table 4 and the graph change trend in Figure 7, under the three different experimental conditions, compared with the $F$ value of the source image, the STFE, GTFE, CLAHE, SSR, MSR, DGR, MSWT, and the 
TABLE 2: Evaluation indicator values of enhanced images under dust and spray.

\begin{tabular}{|c|c|c|c|c|c|}
\hline Evaluation index & Mean & MLMSE & PSNR & MLIE & SSIM \\
\hline Original image $f$ & 39.92 & 31.22 & -- & 1.87 & -- \\
\hline Proposed STFE & 40.01 & 96.49 & 30.49 & 2.01 & 0.97 \\
\hline Proposed GTFE & 39.99 & 89.52 & 30.87 & 1.91 & 0.97 \\
\hline CLAHE & 60.25 & 132.60 & 17.21 & 2.07 & 0.74 \\
\hline SSR & 61.66 & 128.00 & 17.75 & 2.08 & 0.75 \\
\hline MSR & 78.88 & 109.61 & 14.88 & 2.01 & 0.77 \\
\hline DGR & 94.32 & 82.35 & 13.30 & 1.85 & 0.70 \\
\hline CLAHE-DWT & 110.58 & 116.93 & 10.58 & 2.22 & 0.52 \\
\hline Target algorithm & 78.08 & 117.06 & 14.87 & 2.24 & 0.65 \\
\hline
\end{tabular}

TABLE 3: Evaluation indicator values of enhanced images under uneven lighting.

\begin{tabular}{|c|c|c|c|c|c|}
\hline Evaluation index & Mean & MLMSE & PSNR & MLIE & SSIM \\
\hline Original image $f$ & 11.17 & 10.68 & -- & 0.85 & -- \\
\hline Proposed STFE & 11.12 & 23.94 & 37.39 & 0.83 & 0.99 \\
\hline Proposed GTFE & 11.12 & 22.51 & 37.74 & 0.75 & 0.99 \\
\hline CLAHE & 23.66 & 23.75 & 23.00 & 0.90 & 0.71 \\
\hline SSR & 20.75 & 18.44 & 24.26 & 0.82 & 0.77 \\
\hline MSR & 44.90 & 34.60 & 16.52 & 1.05 & 0.33 \\
\hline DGR & 49.55 & 12.38 & 16.23 & 0.87 & 0.41 \\
\hline CLAHE-DWT & 62.71 & 22.52 & 13.32 & 0.90 & 0.28 \\
\hline Target algorithm & 27.53 & 24.89 & 20.91 & 1.10 & 0.61 \\
\hline
\end{tabular}

TABLE 4: Evaluation indicator values of enhanced images.

\begin{tabular}{|c|c|c|c|c|c|}
\hline Evaluation index & Mean & MLMSE & PSNR & MLIE & SSIM \\
\hline Original image $f$ & 45.11 & 95.34 & -- & 1.86 & -- \\
\hline Proposed STFE & 82.51 & 249.06 & 14.07 & 2.09 & 0.64 \\
\hline Proposed GTFE & 82.50 & 251.24 & 14.07 & 2.06 & 0.64 \\
\hline CLAHE & 65.44 & 206.71 & 18.19 & 2.03 & 0.81 \\
\hline SSR & 65.86 & 195.31 & 18.23 & 2.01 & 0.81 \\
\hline MSR & 64.79 & 170.99 & 21.43 & 1.96 & 0.85 \\
\hline DGR & 81.69 & 102.41 & 16.16 & 1.90 & 0.76 \\
\hline CLAHE-DWT & 100.11 & 186.10 & 11.41 & 2.06 & 0.54 \\
\hline Target algorithm & 73.72 & 181.63 & 16.67 & 2.14 & 0.76 \\
\hline
\end{tabular}

TABLE 5: The comprehensive indicator $F$ of different algorithms.

\begin{tabular}{|c|c|c|c|c|c|c|c|c|c|}
\hline Different algorithms & Original image $f$ & Proposed STFE & Proposed GTFE & CLAHE & SSR & MSR & DGR & $\begin{array}{c}\text { CLAHE- } \\
\text { DWT }\end{array}$ & Target algorithm \\
\hline Experiment 1 & 0.20 & 0.66 & 0.60 & 0.62 & 0.60 & 0.65 & 0.48 & 0.52 & 0.68 \\
\hline Experiment 2 & 0.21 & 0.63 & 0.59 & 0.57 & 0.60 & 0.52 & 0.38 & 0.51 & 0.59 \\
\hline Experiment 3 & 0.22 & 0.61 & 0.57 & 0.52 & 0.44 & 0.60 & 0.32 & 0.40 & 0.63 \\
\hline 17 images & 0.20 & 0.61 & 0.59 & 0.60 & 0.57 & 0.57 & 0.37 & 0.48 & 0.63 \\
\hline
\end{tabular}

proposed algorithm is improved by $205 \%, 195 \%, 200 \%$, $185 \%, 185 \%, 85 \%, 140 \%$, and $215 \%$, respectively. Compared with the algorithm of proposed STFE, proposed GTFE, CLAHE, SSR, MSR, DGR, and MSWT, the overall performance indicator of the proposed algorithm is improved by $3.28 \%, 6.78 \%, 5.00 \%, 10.53 \%, 10.53 \%, 70.27 \%$, and $31.25 \%$, respectively. In three typical visual scenarios, compared with the other seven algorithms, the comprehensive performance of the proposed algorithm is better, that is, the proposed algorithm has the best comprehensive enhancement performance and robustness and is more suitable for image enhancement in different environments under mine. 


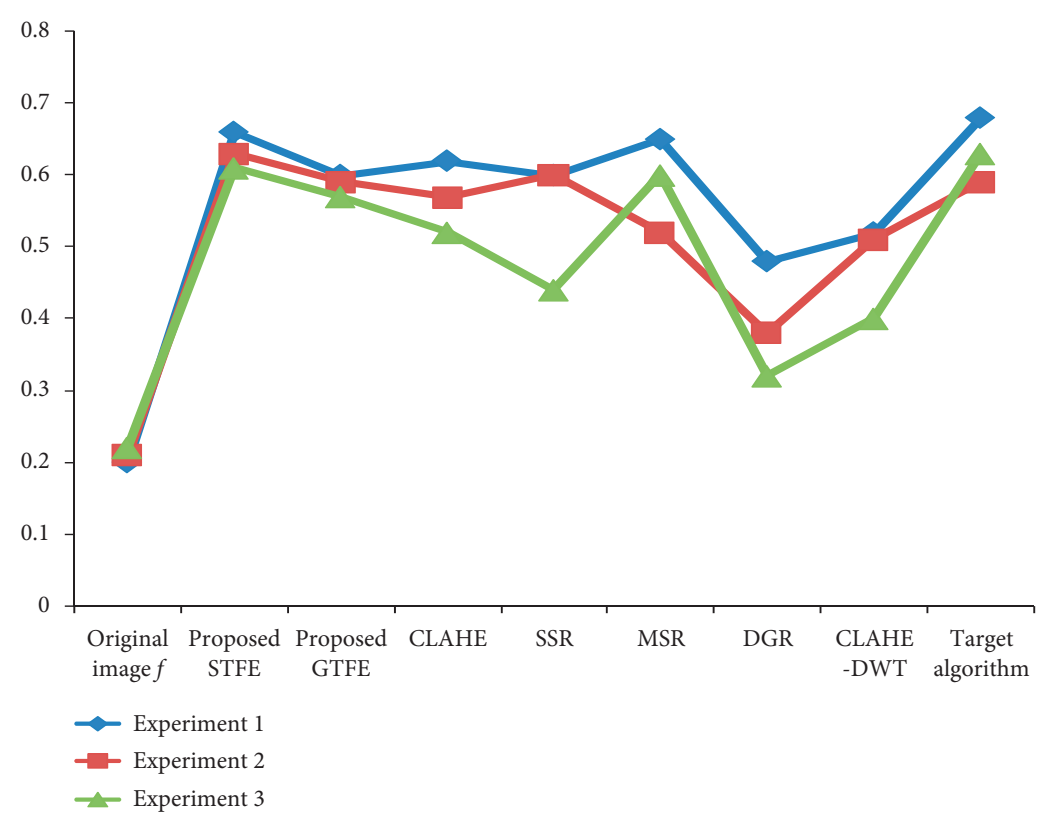

Figure 6: The line chart corresponding to $F$ values of different algorithms.

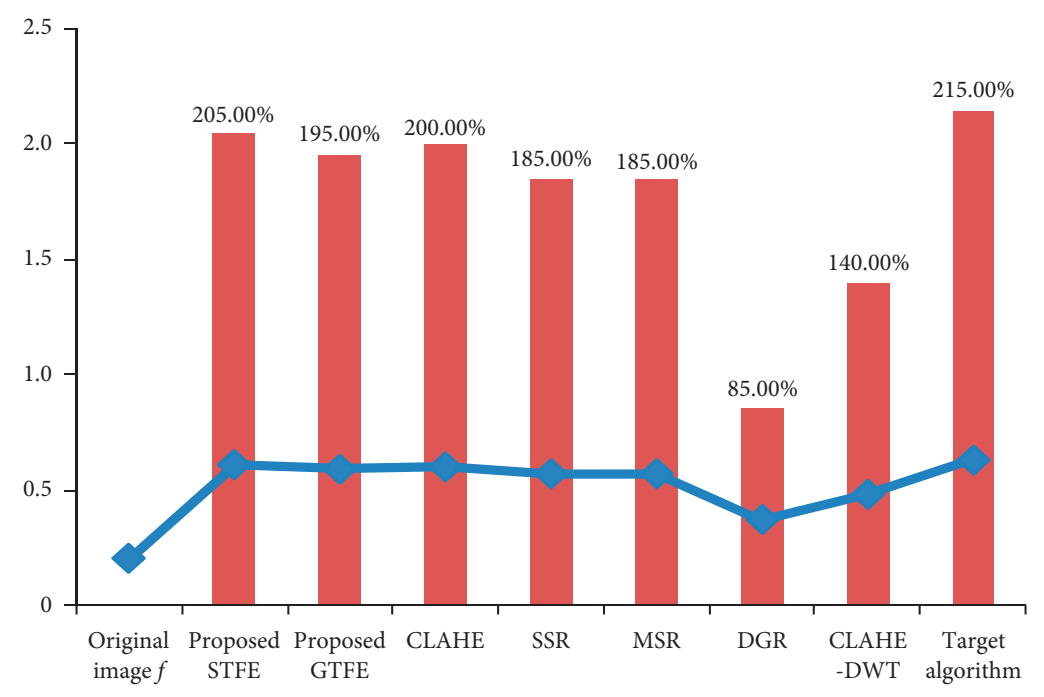

Figure 7: The $(F)$ value of different algorithms and the relative percentage increase compared with the original image.

\section{Conclusions}

The illumination environment in coal mine is complex, such as low illumination, dust and spray, and uneven lighting. These factors will lead to poor visual effects of mine images and are not conducive to the analysis and processing of the images of surveillance videos. In order to meet the practical application in coal mines, this paper proposes an image enhancement algorithm of degraded image enhancement using dual-domain-adaptive wavelet and improved fuzzy transform. A Garrate threshold function with adaptive adjustment factor and enhancement coefficient is designed, and an improved PAL fuzzy enhancement algorithm is constructed. Meanwhile, combining the advantages of DDF,
CLAHE, gamma, Bayesian estimation, and other methods in image enhancement applications, the realization principle of the proposed algorithm is established. Finally, the de-noising and enhancement effects of the proposed algorithm in different environments of the mine are analyzed, and subjective and objective comparison and analysis are carried out with other algorithms.

The results show that the proposed algorithm can improve the overall brightness and contrast of the image, reduce the noise in the image, and make the edge and detail information clearer, whose visual effect is better than other algorithms. Compared with the original degraded image, the comprehensive performance of STFE, GTFE, CLAHE, SSR, MSR, DGR, MSWT, and the proposed algorithm have 
increased by $205 \%, 195 \%, 200 \%, 185 \%, 185.00 \%, 85 \%, 140 \%$, and $215 \%$, respectively. Compared with the algorithm of STFE, GTFE, CLAHE, SSR, MSR, DGR, and MSWT, the comprehensive performance of the proposed algorithm has increased by $3.28 \%, 6.78 \%, 5.00 \%, 10.53 \%, 10.53 \%, 70.27 \%$, and $31.25 \%$, respectively. Under different experimental conditions, the comprehensive enhancement performance of the proposed algorithm is the best, the robustness is the best, and it is more suitable for image enhancement in different environments in underground mines.

\section{Data Availability}

The data used to support the findings of this study are available from the corresponding author upon request.

\section{Conflicts of Interest}

The authors declare that they have no conflicts of interest.

\section{Acknowledgments}

This research was funded by the National Key Research \& Development Program of China (grant no. 2017YFC0804302) and the National Key Research \& Development Program of China (grant no. 2016YFC0801800).

\section{References}

[1] J. P. Sun, "Technologies of monitoring and communication in the coal mine," Journal of China Coal Society, vol. 35, no. 11, pp. 1925-1929, 2010.

[2] G. F. Wang, F. Liu, Y. H. Pang et al., "Coal mine intellectualization: the core technology of high quality development," Journal of China Coal Society, vol. 44, no. 2, pp. 349-357, 2019.

[3] Z. Q. Xu, Z. Q. Lv, W. D. Wang et al., "Machine vision recognition method and optimization for intelligent separation of coal and gangue," Journal of China Coal Society, vol. 45, no. 6, pp. 2207-2216, 2020.

[4] F. Zhang, X. H. Sun, and D. L. Cui, "Method of tracking and positioning for mobile target based on ORB features and binocular vision in mine," Journal of China Coal Society, vol. 43, no. S2, pp. 654-662, 2018.

[5] W. Zhang and G. Zhang, "Image feature matching based on semantic fusion description and spatial consistency," Symmetry, vol. 10, no. 12, p. 725, 2018.

[6] H. Han, P. F. Wang, R. H. Liu et al., "Experimental study on atomization characteristics and dust-reduction performance of four common types of pressure nozzles in underground coal mines," International Journal of Coal Science \& Technology, vol. 7, 2020.

[7] N. Zhi, S. J. Mao, and M. Li, "Enhancement algorithm based on illumination adjustment for non-uniform illuminance video images in coal mine," Journal of China Coal Society, vol. 42, no. 8, pp. 2190-2197, 2017.

[8] L. Si, Z. Wang, R. Xu, C. Tan, X. Liu, and J. Xu, "Image enhancement for surveillance video of coal mining face based on single-scale retinex algorithm combined with bilateral filtering," Symmetry, vol. 9, no. 6, p. 93, 2017.

[9] D. W. Wang, P. F. Han, J. L. Fan et al., "Multispectral image enhancement based on illuminance-reflection imaging model and morphology operation," Acta Physica Sinica, vol. 67, no. 21, pp. 104-114, 2018.
[10] S. LiuY. Zhang et al., "Automatic segmentation of dermoscopy images using saliency combined with adaptive thresholding based on wavelet transform," Multimedia Tools and Applications, vol. 79, no. 21, pp. 14625-14642, 2020.

[11] S. Juneja and R. Anand, "Contrast enhancement of an image by DWT-SVD and DCT-SVD," Advances in Intelligent Systems and Computing, vol. 542, 2017.

[12] S. Satapathy, V. Bhateja, K. Raju, and B. Janakiramaiah, Data Engineering and Intelligent Computing, pp. 595-603, Springer, Singapore, 2018.

[13] F. Kallel and A. Ben Hamida, "A new adaptive gamma correction based algorithm using DWT-SVD for non-contrast CT image enhancement," IEEE Transactions on Nanobioscience, vol. 16, no. 8, pp. 666-675, 2017.

[14] M. Zhou, K. Jin, S. Wang, J. Ye, and D. Qian, "Color retinal image enhancement based on luminosity and contrast adjustment," IEEE Transactions on Biomedical Engineering, vol. 65, no. 3, pp. 521-527, 2018.

[15] H. Singh, A. Kumar, L. K. Balyan, and G. K. Singh, "Swarm intelligence optimized piecewise gamma corrected histogram equalization for dark image enhancement," Computers \& Electrical Engineering, vol. 70, no. 8, pp. 462-475, 2018.

[16] S. K. Karan and S. R. Samadder, "Improving accuracy of longterm land-use change in coal mining areas using wavelets and Support Vector Machines," International Journal of Remote Sensing, vol. 39, no. 1, pp. 84-100, 2018.

[17] C. Yang, M. Zhang, Z. Zhang, L. Wei, R. Chen, and H. Zhou, "Non-rigid point set registration via global and local constraints," Multimedia Tools and Applications, vol. 77, no. 24, pp. 31607-31625, 2018.

[18] C. Bae, Y. Y. Chung, and J. Lee, "Image based video querying algorithm using 3-level Haar wavelet transform features," in Proceedings of the 8th International Conference on Computer Science and its Applications(CSA), pp. 779-785, Springer Singapore, Bangkok, Thailand, December 2016.

[19] S. Lei, M. M. Lu, J. Q. Lin et al., "Remote sensing image denoising based on improved semi-soft threshold," Signal, Image And Video Processing, vol. 15, 2020.

[20] A. Vishwakarma, M. K. Bhuyan, and Y. Iwahori, "Nonsubsampled shearlet transform-based image fusion using modified weighted saliency and local difference," Multimedia Tools and Applications, vol. 77, no. 24, pp. 32013-32040, 2018.

[21] L. X. Zhang, Y. L. Wang, X. Y. Deng et al., "Wavelet Semi-soft threshold denoising based on ensemble empirical mode decomposition," Journal of Detection \& Control, vol. 40, no. 5, pp. 53-57, 2018.

[22] K. K. Il, R. U. Hwan, C. B. Pil et al., “An appropriate thresholding method of wavelet denoising for dropping ambient noise," International Journal of Wavelets, Multiresolution and Information Processing, vol. 16, no. 3, Article ID 1850012, 2017.

[23] F. He and X. He, "A continuous differentiable wavelet shrinkage function for economic data denoising," Computational Economics, vol. 54, no. 2, pp. 729-761, 2019.

[24] W. Q. Fan and Y. Liu, "Fuzzy enhancement algorithm of coal mine degradation image based on adaptive wavelet transform," Journal of China Coal Society, vol. 45, no. 12, pp. 4248-4260, 2020.

[25] S. Saravani, R. Shad, and M. Ghaemi, "Iterative adaptive despeckling SAR image using anisotropic diffusion filter and Bayesian estimation denoising in wavelet domain," Multimedia Tools and Applications, vol. 77, no. 23, pp. 3146931486, 2018. 
[26] Y. Y. Sha, L. X. Xi, X. G. Zhang et al., "Application of wavelet threshold denoising in PMD measurement by fixed analyzer method," in Proceedings of the 10th International Conference on Information Optics and Photonics, Article ID 109644S, Beijing, China, July 2018.

[27] N. Chetih, Z. Messali, A. Serir et al., "Robust fuzzy c-means clustering algorithm using non-parametric Bayesian estimation in wavelet transform domain for noisy MR brain image segmentation," Iet Image Processing, vol. 12, no. 5, pp. 652660, 2017.

[28] D. R. Sachin and D. D. Dharmpal, "Image denoising with modified wavelets feature restoration," International Journal of Computer Science Issues, vol. 9, no. 2, pp. 403-412, 2012.

[29] P. Tang and B. P. Guo, "Wavelet denoising based on modified threshold function optimization method," Journal of Signal Processing, vol. 33, no. 1, pp. 102-110, 2017.

[30] Y. H. Huo and W. Q. Fan, "Face recognition method under complex light conditions in coal mines," Laser \& Optoelectronics Progress, vol. 56, no. 1, Article ID 011003, 2019.

[31] L. Zhuang and Y. Guan, "Image enhancement using modified histogram and log-exp transformation," Symmetry, vol. 11, no. 8, p. 1062, 2019.

[32] P. Naveen and P. Sivakumar, "Adaptive morphological and bilateral filtering with ensemble convolutional neural network for pose-invariant face recognition," Journal of Ambient Intelligence and Humanized Computing, 2021.

[33] H. D. Sun, "Wavelet core selection to mine monitoring image denosing algorithm," Coal Mine Machinery, vol. 35, no. 11, pp. 271-272, 2014.

[34] A. Zotin, "Fast algorithm of image enhancement based on multi-scale retinex," Procedia Computer Science, vol. 131, no. 4, pp. 6-14, 2018.

[35] N. Zhi, S. J. Mao, and M. Li, “An enhancement algorithm for coal mine low illumination images based on bi-gamma function," Journal of Liaoning Technical University (Natural Science), vol. 37, no. 1, pp. 191-197, 2018.

[36] L. D. Huang, W. Zhao, J. Wang et al., "Combination of contrast limited adaptive histogram equalisation and discrete wavelet transform for image enhancement," Iet Image Processing, vol. 9, no. 10, pp. 908-915, 2015.

[37] A. Kaur and C. Singh, "Contrast enhancement for cephalometric images using wavelet-based modified adaptive histogram equalization," Applied Soft Computing, vol. 51, no. 2, pp. 180-191, 2017. 